\title{
Assessing Health System Factors Affecting Access and Delivery of IPTp-SP and ITN to Pregnant Women Attending ANC Clinics in Ghana-A Quantitative Study
}

Virtue Fiawokome De-Gaulle ( $\square$ nunana2009@live.com )

Université de Yaoundé I

\section{Pascal Magnussen}

University of Copenhagen

Joseph Kamgno

Université de Yaoundé I

Wilfred Mbacham

Université de Yaoundé I

Verner Ndudiri Orish

University of Health and Allied Science

Harry Tagbor

University of Health and Allied Science

\section{Research Article}

Keywords: Health System, Intermittent Preventive Treatment (IPT), Insecticide Treated Nets (ITNs), Pregnant woman, Antenatal clinic (ANC), Ghana

Posted Date: April 21st, 2021

DOl: https://doi.org/10.21203/rs.3.rs-424064/v1

License: (a) (1) This work is licensed under a Creative Commons Attribution 4.0 International License. Read Full License 


\section{Abstract}

Introduction: Malaria interventions including use of Sulfadoxine-Pyrimethamine as Intermittent Preventive Treatment (IPTp-SP) and distribution of Insecticide Treated Nets (ITNs) have been implemented through antenatal clinic (ANC) services in Ghana. Yet, the high ANC attendance is not commensurate with the uptake of these interventions, with missed opportunities to deliver the interventions. This study sought to assess the health system factors affecting access and delivery of IPTp-SP and ITN as defined by the Ghana Malaria Policy Guideline to eligible pregnant women attending ANC clinic sessions.

Methods: A quantitative cross-sectional study was conducted in the Volta Region of Ghana, with data collected across three levels of health care delivery facilities, including hospitals, health centres and Community-Based Health Planning Service (CHPS) compounds. Data collection included structured observation checklists to document the communication and interaction between the ANC health staff and pregnant women. Additionally, structured questionnaires were used to elicit information on cadre, trainings attended, knowledge and delivery practices of health workers on IPTp-SP and ITN. Stata 16 was used for data analysis, and a defined delivery algorithm was used to compute appropriate and inappropriate delivery practices, using the Ghana policy directive as a guide. Predictors of appropriate delivery were determined using logistic regression analysis.

Results: Approximately $97 \%$ of the 680 ANC observations had complete information for analysis. Of these, $78 \%$ (511) were eligible for IPTp-SP after excluding women who have less than 16 weeks of gestation, G6PD deficient, malaria positive and have taken 5 doses of IPTp-SP prior to day of observation. Appropriate delivery of IPTp-SP was $76 \%$ (390). Despite the availability of SP, $15 \%$ (75) of all eligible women were not offered the medication and $37 \%$ (44) of inappropriate delivery was due to stock out. ITNs were appropriately delivered to 59\% (139) out of 237 eligible women. Thirty-two percent (77) of eligible women, mostly continuing ANC clients, were not given ITN despite stock availability.

Conclusions: IPTp-SP was appropriately delivered to $76 \%$ of clients as compared to $59 \%$ in the case of ITN. Despite stock availability, IPTp-SP and ITN were not delivered to some eligible women. This highlights health worker related factors influencing delivery.

\section{Background}

Malaria, an infectious parasitic disease, transmitted by mosquitoes continues to be a grave public health problem for the African continent (1). In 2019, 94\% (215 million) of all malaria cases and deaths $(384,000)$ were recorded in the continent, with most of the infections due to Plasmodium (P.) falciparum, the most lethal of the parasites (2). Although everybody is at risk of the disease in endemic regions, children under five years old and pregnant women are the most vulnerable (3).

For pregnant women, their increased vulnerability has been attributed to reduced immunity arising from immunological, hormonal and physiological changes (4) (5). For instance, physiologically, increased blood flow to the skin during pregnancy results in heat dissipation, and this facilitates the release of volatile substances from the skin surface, making it easy for mosquitoes to locate pregnant women (6). In 2019, the World Health Organization (WHO) estimated that out of 33.2 million pregnancies that occurred in sub-Saharan Africa (SSA), approximately 11.6 million of them were exposed to malaria infection (3), and this resulted in about 822,000 low birth weighted (LBW) babies, half of whom were born in the West African sub region (2). In Ghana, about 
370,514 pregnancies are estimated to be exposed to malaria parasites annually, and this results in 30,611 LBW babies (7). Malaria in pregnancy (MiP), mainly $P$. falciparum infection is associated with a number of adverse effects including maternal death, maternal anaemia, miscarriage, still birth, intra-uterine growth restriction and LBW (8) (9) (10). The severity of the effects of the disease in pregnancy is however dependent on the level of acquired immunity (4). In low or unstable malaria transmission areas, with low levels of acquired immunity, pregnant women are more likely to progress towards clinical cases, with untreated persons developing severe disease and even death (4) (5) (11). In moderate and high transmission settings like most parts of SSA, malaria infection during pregnancy is predominantly asymptomatic due to acquired immunity, arising from recurrent infections (12) (13). Nonetheless, the parasites may be present in the placenta and contribute to maternal anaemia (14) (15) which can lead to LBW (16) (17), an important contributor to infant mortality (18).

To mitigate the adverse effects associated with MiP, WHO developed a policy framework with a three pronged approach for malaria control among pregnant women in the African region (19) This entailed (1) administering Intermittent Preventive Treatment using Sulfadoxine-Pyrimethamine (IPTp-SP), starting as early as possible in the second trimester, with monthly intervals for subsequent doses and delivered under Directly Observed Therapy (DOT); (2) distribution and counselling on use of Insecticide Treated Nets (ITNs); (3) with the third being Prompt Case Management of clinical malaria (19). In 2012, evidence from a meta-analysis on higher mean birth weight and less placental malaria from the uptake of three or more IPTp-SP led to WHO recommending at least three doses of IPTp-SP during pregnancy to ensure optimal protection (20). These interventions have been incorporated into the ANC services, thus, IPTp-SP and ITN are given to eligible pregnant women during their scheduled ANC clinic visits (21). Financial support from donor agencies including the Global Fund (22) (23) and local funding from governments of African countries have eased the financial burden on procurement of these preventive interventions, so that IPTp-SP and ITNs are to be given to pregnant women free of charge. With the implementation of these intervention at ANC clinics, it was expected that the high ANC attendance in most SSA countries would translate into comparable uptake of the interventions, resulting in substantial reduction in LBW babies. Unfortunately, this has not been the case, with huge disparities between ANC attendance and IPTp-SP uptake and ITN ownership recorded (24) and thousands of LBW babies attributable to malaria born in the region annually (3). In 2016, the World Malaria Report (WMR) indicated that only 39\% of pregnant women who attended ANC received an ITN (25), and in 2018, only $31 \%$ of pregnant women received IPTp-SP3 (3).

In Ghana, national estimates from the 2014 Demographic and Health Survey (GDHS) revealed that $87 \%$ of pregnant women attended four or more ANC visits, with most of them starting in their second trimester, but only $39 \%$ reported taking three or more doses of SP (26). Whilst the uptake of these interventions could be influenced by individual factors such as pregnant woman's acceptance or refusal to take the intervention based on personal, cultural or religious beliefs (27), health system factors directly linked to delivery of the interventions have been cited as a possible major cause of this disparity (28) (24) (Hill et al., 2013; Andrews et al., 2015). Although some studies have been conducted in Ghana on IPTp-SP and ITN, they have been limited to assessing pregnant women factors influencing uptake (29) (30) (31). Not much has been done on assessing the factors related to the health system's effect on delivery of IPTp-SP and ITN. This study therefore sought to assess the delivery appropriateness of IPTp-SP and ITN to pregnant women attending ANC clinic sessions, using the Ghana MiP policy directive as a guide, and also ascertain health system factors influencing delivery of the two preventive interventions. 


\section{Methods}

\section{Study Site}

This study was conducted in the Agortime-Ziope and South Tongu Districts located in the Volta Region of Ghana. The Agortime-Ziope district is predominantly rural, with a population of about $35,360,49 \%(17,326)$ of whom are females (32). Approximately, 39.4\% $(6,843)$ of the females are between $15-49$ years, and the General Fertility Rate (GFR), defined as the number of live births per 1,000 women aged between 15-49 years in a given year was 109.2 according to the 2010 Census (32). The district had no hospital at the time of the data collection, however, there were three health centres, four Community-Based Health Planning Services (CHPS) compound, and one private maternity home (32). The South Tongu district is peri-urban with a population of $110,777,52 \%$ of whom are females (33). Approximately, $37 \%(21,325)$ of the females are aged between $15-49$ years and the GFR (103.3) is comparable to that of Agortime-Ziope. (33). The district has a total of 29 health facilities (two hospitals, four health centres, 18 CHPS compounds and 5 private clinics) (33). In each district data was collected across three levels of healthcare delivery facilities - hospitals, health centres and CHPS compound. The hospitals provide both primary and secondary healthcare services and serve as referral centres for the health centres and the CHPS compounds who provide primary healthcare services.

\section{Study Population}

The study population comprised of pregnant women and ANC health staff. Specifically, all pregnant women irrespective of gestational age attending ANC clinic during the study period were eligible to participate in the study. With regards to the ANC health staff, a complete enumeration of all ANC staff who provide clinical care in the study facilities was carried out.

\section{Sample Size Calculation}

A sample size of 680 was calculated using Cochran's formula $\left(n=z^{2} p(1-p) / e^{2}\right)$ for calculating sample sizes for cross sectional studies. Where " $n$ "is the computed sample size, " $z$ " the desired confidence interval at $95 \%$ ( $z=$ 1.96), " $\boldsymbol{p}$ "the estimated proportion of an attribute that is present in the population, and " $\boldsymbol{e}$ "the desired level of precision at $5 \%$ (34). To determine the most appropriate prevalence " $\mathrm{p}$ " of the two interventions (IPTp-SP or ITN) to use, the most current available national estimates of IPTp-SP3 (36.7\%) (35) and ITN use (43\%) (36) at the time of the data collection period was used. ITN use generated the largest sample size of 377 for each study district (Table 1). The Cochrane's finite population correction formula (34) was then used to correct the sample sizes for each district to reflect a feasible and representative number of pregnant woman that can be sampled, after which a $10 \%$ non-response or incomplete dataset rate was computed (Table 1). This percentage was informed by a Malawian study on IPTp-SP, where $8 \%$ of the data were incomplete, thus excluded from analysis (37). The number of pregnant women who access ANC services in the various health facilities vary, therefore a proportionate method was used to determine the percentage of pregnant women to be sampled in each facility in a particular district. The was determined by making the total number of annual ANC registrants (reference year is 2018 retrieved from the District Health Management Information system (DHIMS), in each health facility a fraction of the sum of all pregnant women registered for ANC in all the participating health facilities in each district annually. This fraction was then multiplied by the calculated sample size for each district to get the proportion of pregnant women to be sampled from each facility (Table 1). 
Table 1: Sample Size Calculation 
Sample size calculation

Prevalence of MiP Interventions in Ghana

IPTp-SP3

$36.7 \%$

$n=\frac{z^{2} p(1-p)}{e^{2}}$

Sample size to be used for each district
ITN Use

$43 \%$

377

Finite population correction for computed sample size

Agortime-Ziope

Computed Cochrane sample size $\left(\mathrm{n}_{\mathrm{o}}\right)$

Population of ANC Registrants by district in $2018(\mathrm{~N})$

$$
n=\frac{n_{0}}{1+\frac{\left(n_{0}-1\right)}{N}}
$$

Adding a $10 \%$ non response rate (Final sample size)
377

1078

280

308
372

South-Tongu

377

3209

338

Proportional allocation of study population to be sampled in health facilities

\begin{tabular}{|c|c|c|c|c|}
\hline Districts & Health facilities & $\begin{array}{l}\text { Yearly, ANC } \\
\text { registrants (Ni) }\end{array}$ & $\begin{array}{l}\text { Proportion of registrants } \\
\text { wi =Ni / N }\end{array}$ & $\begin{array}{l}\text { Sample per } \\
\text { facility } \\
\text { ni= wi*n }\end{array}$ \\
\hline \multirow[t]{5}{*}{$\begin{array}{l}\text { South } \\
\text { Tongu }\end{array}$} & $\begin{array}{l}\text { Sogakope District } \\
\text { Hospital }\end{array}$ & 944 & 0.41 & 153 \\
\hline & $\begin{array}{l}\text { Camboni Catholic } \\
\text { Hospital }\end{array}$ & 931 & 0.41 & 151 \\
\hline & Dabala Health Centre & 257 & 0.11 & 42 \\
\hline & $\begin{array}{l}\text { Agbakope CHPS } \\
\text { Compound }\end{array}$ & 160 & 0.07 & 26 \\
\hline & Total (N) & 2292 & 1 & 372 \\
\hline \multirow{3}{*}{$\begin{array}{l}\text { Agortime } \\
\text { Ziope }\end{array}$} & Kpetoe Health Centre & 544 & 0.58 & 180 \\
\hline & Ziope Health Centre & 336 & 0.36 & 112 \\
\hline & $\begin{array}{l}\text { Akpokope CHPS } \\
\text { Compound }\end{array}$ & 51 & 0.05 & 16 \\
\hline
\end{tabular}




\section{Data Collection Procedure}

The study design was cross-sectional using quantitative methods. Seven health facilities across three levels of care were purposively sampled. This sampling was done to help identify the health system factors peculiar to each level of care, and ascertain how that affects the delivery of IPTp-SP and ITN. In the South Tongu District, the district hospital, one health centre and CHPS compound with the highest number of ANC clients compared to the other CHPS and health centres in that district were included. Additionally, a faith-based facility comparable to the level of a district hospital was also included to provide an insight into the delivery practices peculiar to nongovernmental health facilities. In the Agortime-Ziope District, there was no district hospital at the time of data collection, two health centres; one in an urban and the other in a peri-urban settlement were included, in addition one CHPS compound with the highest number of ANC clients was sampled. Data collection tools used included observation checklist and questionnaires. Two different observation checklists were used, one was used to document the interaction between ANC health staff and pregnant women and the second to document general ANC activities on a daily basis, including stock of IPTp-SP and ITN. Four community health nurses were recruited as research assistants (RAs) and trained on the study aim, data collection methods and research ethics for two days, followed by one day field work. Prior to commencing data collection, a meeting was convened in each study facility with the health workers to inform them about the study and also seek their consent to be observed, especially the ANC staff. Data collection commenced with all the consented ANC health staff interviewed with a questionnaire. The questionnaire elicited information on cadre, trainings attended and knowledge on the Ghana Policy on IPTP-SP and ITN. Consecutive sampling, a technique in which every study population meeting the inclusion criteria is sampled until the sampled size is reached (38) was employed in the sampling of pregnant women. The RAs approached pregnant women as they awaited their turn to be attended to, introduced the study to them and obtained written consent from those willing to participate. The observation of the ANC consultation sessions were then carried out, with the actions and communications between pregnant women and health staff documented. Data collected included the cadre of health worker delivering care, gestational age of pregnant woman, number of ANC sessions attended, physical examinations and tests conducted by the ANC staff, medications and treatments administered including IPTp-SP, and delivery of ITN. Data was collected on every ANC clinic day till the required sample size was obtained. All Filled data collection tools were cross-checked on a daily basis for data accuracy and completeness by a field supervisor.

\section{Study Definitions}

Using the 2014 Ghana MiP Policy directive (39), an algorithm to assess the delivery of IPTp-SP and ITN in the ANC unit was developed. The policy directive specified that SP should be administered as a single dose of three tablets of $500 \mathrm{mg}$ Sulfadoxine and $25 \mathrm{mg}$ pyrimethamine, commenced from 16 weeks of gestation or at quickening, to pregnant women who are not clinically diagnosed with malaria or G6PD deficieny. The medication should also be administered under Directly Observed Therapy (DOT) with monthly intervals. While, up to seven doses of SP can be taken as specified in the policy document, delivery in all the health facilities across Ghana has been capped at five doses. The defined algorithm for appropriate delivery of IPTp-SP for this study was therefore defined based on successful completion of three steps, which were: 
i. Eligible pregnant women ( $\geq 16$ weeks, not clinically ill with malaria, not G6PD deficient and taken $<5$ doses of SP) being offered IPTp-SP by ANC health staff, and

ii. Administered under DOT and

iii. Informed her next ANC visit either verbally or written in their maternal health record book (MHRB) to foster continuity of the medication.

Inappropriate delivery of IPTp-SP entailed any of the actions or inactions below by the health worker:

i. Health worker not offering IPTp-SP to eligible pregnant women or,

ii. Health worker offering IPTp-SP, but not administering under DOT coupled with telling the pregnant women her next visit or not.

iii. Health worker offering IPTp-SP, under DOT, but not telling the pregnant women her next visit, verbally or writing it in her MHRB.

Similarly, delivery of ITN was assessed by an algorithm. In Ghana, every pregnant woman attending ANC clinic is eligible for an ITN free of charge. Hence, eligibility for ITN was defined as any pregnant woman who has not yet been given an ITN since starting ANC. Appropriate delivery of ITN was therefore defined as:

i. Eligible pregnant women being given an ITN during ANC visit.

Inappropriate delivery was defined as:

i. Eligible pregnant woman not being given an ITN during ANC observation.

\section{Data Analyses}

Data were entered and cleaned using Statistical Package for Social Sciences (SPSS) (SPSS Inc., Chicago, IL) version 22, after which the data was imported into STATA 16 SE software (Stata Corp LP) for analysis.

Continuous variables in the observation checklist such as gestational age of pregnancy and number of ANC sessions attended were reclassified into ranges. Prior to computing IPTp-SP delivery, ineligible pregnant women ( $<16$ weeks, G6PD deficient, malaria positive and those who have taken already taken 5 doses of IPTp-SP) were filtered from the overall sample of pregnant women observed (Figure 1). This gave rise to a sub-sample of pregnant women, which was then used to assess the delivery of IPTp-SP (Figure 2). Similarly, ineligible pregnant women (have already received ITN during that pregnancy) were filtered out before the delivery of ITN was computed (Figure 3). To ensure accurate assessment of the factors influencing the delivery of both IPTp-SP and ITN, inappropriate delivery that occurred during periods of stock out was filtered out, in order not to confound estimation of other predictive variables. Pearson Chi-Square $\left(\mathrm{X}^{2}\right)$ and Fishers Exact Test analysis were used to determine the association between selected explanatory variables and delivery of IPTp-SP and ITN. Statistical significance was set at a p-value of less than $0.05(p<0.05)$. To ascertain the strength of association between appropriate delivery of the interventions and significant explanatory variables from the $\mathrm{X}^{2}$ analysis, a bivariable logistic regression analysis was performed by estimating Odds Ratios (ORs) with 95\% confidence intervals (Cls). All explanatory variables with $\mathrm{p}<0.05$ were fitted into an investigator led backward-stepwise multivariable logistic regression model to further ascertain the strength of association with the outcome variable by estimating the 
adjusted Odds Ratios (aORs). P-value of $<0.05$ was deemed significant. The results of the study are presented in figures and tables.

\section{Results}

\section{Characteristics of Health Facilities, ANC Units, Pregnant Women}

Seven health facilities across three levels of health care delivery system were included in this study. All the health facilities provided ANC services, however Focused ANC (FANC), which is an individualised, client centred, comprehensive care, with emphasis on disease detection rather than risk assessment (40) was limited to the hospitals and CHPS compounds. Although health centres had laboratory services, Glucose-6-phosphate dehydrogenase (G6PD) tests were conducted only in the hospital laboratories. MiP and case management guidelines were unavailable in some of the facilities, however, wall charts on IPTp-SP and ITN were visibly displayed on the walls in all the ANC units. Stock outs of SP and ITNs were recorded during the data collection period in some of the health centres (Table 2).

\section{Organisation and Delivery of ANC Services}

Owing to the free maternal health care policy in Ghana, all pregnant women are entitled to free enrolment on the National Health Insurance Scheme (NHIS) to enable access to maternal health services. In the hospitals and health centres, a section outside the ANC consulting room was used for recording blood pressure, temperature, weight and height of the pregnant women. A waiting area was also designated for pregnant women to sit and wait their turn, with collective health education organized for them. The hospitals, having two ANC consulting rooms with assigned midwives, practiced FANC. The health centres, having just one ANC consulting room with midwives alternating when on duty did not practice FANC. Services provided to pregnant women in the ANC consulting room included; history taking, physical examination (palpating the abdomen, measuring fundal height, and listening to foetal heartbeat), Point of Care (POC) tests (HIV tests, urine dipstick for protein and sugar, and malaria RDT), administration of treatments (IPTp-SP) and immunizations (Tetanus), case management of some disease conditions (E.g. uncomplicated malaria), distribution of ITN, and health education.

\section{Table 2. Characteristics of Health Facilities and their ANC Units}




\begin{tabular}{|c|c|c|c|c|c|c|c|}
\hline \multirow[b]{2}{*}{ Characteristics } & \multicolumn{2}{|l|}{ Hospitals } & \multicolumn{3}{|c|}{ Health Centres } & \multicolumn{2}{|c|}{ CHPS Compounds } \\
\hline & HOSP 1 & HOSP 2 & $\mathrm{HC} 1$ & HC 2 & $\mathrm{HC} 3$ & CHPS 1 & CHPS 2 \\
\hline \multicolumn{8}{|l|}{ Health personnel } \\
\hline Facility in-charge & Physician & Physician & $\mathrm{PA}[1]$ & PA & PA & Midwife & Midwife \\
\hline Pharmacist & Yes & Yes & No & No & No & No & No \\
\hline \multicolumn{8}{|l|}{ Maternity services } \\
\hline ANC services & Yes & Yes & Yes & Yes & Yes & Yes & Yes \\
\hline Skilled delivery & Yes & Yes & Yes & Yes & Yes & Yes & Yes \\
\hline Post-natal & Yes & Yes & Yes & Yes & Yes & Yes & Yes \\
\hline \multicolumn{8}{|l|}{ Laboratory and scan } \\
\hline Functioning laboratory & Yes & Yes & Yes & Yes & No & No & No \\
\hline Ultrasound scan & Yes & Yes & No & No & Yes & No & Yes \\
\hline \multicolumn{8}{|l|}{ Medical storage space } \\
\hline Pharmaceutical storage & Yes & Yes & No & No & No & No & No \\
\hline Non- pharmaceutical & Yes & Yes & No & No & No & No & No \\
\hline Combined storage & No & No & Yes & Yes & Yes & Yes & Yes \\
\hline \multicolumn{8}{|l|}{ The ANC Units } \\
\hline \multicolumn{8}{|l|}{ Health Personnel } \\
\hline No. of midwives at ANC & 3 & 3 & 2 & 3 & 2 & 1 & 2 \\
\hline Auxiliary nurses & 1 & 4 & 0 & 0 & 0 & 2 & 2 \\
\hline Student[2] or Rotation nurses[3] & 6 & 0 & 1 & 1 & 2 & 0 & 1 \\
\hline \multicolumn{8}{|l|}{ Type of ANC } \\
\hline FANC & Yes & Yes & No & No & No & Yes & Yes \\
\hline \multicolumn{8}{|l|}{ Laboratory Tests/P.O.C } \\
\hline G6PD Test & Yes & Yes & No & No & $N A[4]$ & NA & NA \\
\hline $\mathrm{BF}[5]$ for malaria & Yes & Yes & Yes & Yes & NA & NA & NA \\
\hline Malaria RDT & Yes & Yes & Yes & Yes & Yes & Yes & Yes \\
\hline \multicolumn{8}{|l|}{ Malaria Guidelines } \\
\hline MIP guidelines & Yes & Yes & No & No & Yes & No & Yes \\
\hline Case management & Yes & Yes & No & No & Yes & Yes & Yes \\
\hline Malaria Wall Charts & & & & & & & \\
\hline
\end{tabular}




\begin{tabular}{|lccccccc|} 
IPTp-SP & Yes & Yes & Yes & Yes & Yes & Yes & Yes \\
\hline ITN & Yes & Yes & Yes & Yes & Yes & Yes & Yes \\
\hline MIP Effects & No & No & Yes & No & No & No & No \\
\hline ANC session observations & & & & & & & \\
\hline No. of observation & 153 & 137 & 179 & 111 & 39 & 15 & 23 \\
\hline Stock of SP and ITN & & & & & & & \\
\hline Stock out of SP & No & No & Yes & No & Yes & No & No \\
\hline Stock out of ITN & Yes & No & Yes & Yes & No & No & No \\
\hline
\end{tabular}

[1] Physician Assistant

[2] Nursing students on clinical practical

[3] Newly graduated nurses serving for one year being assigned to health facilities

[4] Not Available

[5] Blood film

\section{Determining Eligibility of Pregnant Women for IPTp-SP}

Six hundred and eighty observations of the ANC clinic sessions between pregnant women and ANC staff were conducted. Some of the observations had pertinent missing data such as current gestational age, and this was mainly due to unfilled sections in the MHRB. Incomplete records were omitted from the analysis. After data cleaning, 657 (96.6\%) of the observed cases had complete datasets tools adequate for analysis. Ineligible women as specified in the Ghana MiP policy document were excluded from the analysis, with the derived subsample of pregnant women eligible for IPTp-SP being 511. (Figure 1).

\section{Assessment of IPTp-SP Delivery}

Using the study's defined delivery assessment algorithm, appropriate and inappropriate delivery of IPTp-SP was computed. Figure 2, shows that out of the 511 pregnant women eligible for IPTp-SP, 76.7\% (392) were offered the medication by the ANC staff, of which 390 were administered under DOT, with all of them being told their next ANC visit date. IPTp-SP was appropriately administered to $76.3 \%$ of the pregnant women. On the other hand, $23.3 \%$ (119) of eligible pregnant women were not offered the medication, $37 \%$ (44) of which occurred during a period of stock out. Despite the availability of SP in health facilities during the data collection period, almost $15 \%$ (75) of all eligible women were not offered the medication.

\section{Factors Influencing Delivery of IPT-SP}

To ensure accurate assessment of the factors influencing delivery of IPTp-SP, inappropriate delivery during periods of stock out were filtered out from the denominator of eligible pregnant women. The results from the 
Pearson Chi-Square analysis showed that years of experience of the ANC staff was an important factor influencing delivery of IPTp-SP ( $p<0.007)$. Health workers who had 1-5years experience delivered IPTp-SP appropriately to $88.9 \%$ (152) of pregnant women. Type of pregnant woman (first time or continuing ANC client) was found to be significant factor $(\mathrm{p}<0.0001)$ affecting delivery of IPTp-SP, with $86.1 \%(360)$ of the continuing pregnant women having IPTp-SP delivered to them appropriately. The number of ANC sessions attended by pregnant women $(p<0.0001)$ and gestational age of pregnancy $(p<0.0001)$ were also significantly associated with the delivery of IPTp-SP (Table 3).

\section{Table 3: Factors Influencing Delivery of IPT-SP}


Delivery of IPTp-SP When in Stock

$(\mathrm{N}=467)$

\section{Factors}

\section{Type of facility}

District hospital

Faith-based hospital

Health Centres

CHPS Compounds

\section{Ultrasound scan}

Available

Unavailable

\section{Type of worker}

Midwife

Rotation nurse

ANC years of experience

$<$ one year

1-5years

$>5$ years

MiP Training

Yes

No

\section{Can SP be taken on an empty stomach}

Yes (Correct)

No (Incorrect)

IPTp-SP can be given at 16 weeks of gestation irrespective of quickening

Yes (Correct)

No (Incorrect)

Type of Pregnant woman

First time ANC

Continuing ANC
Appropriate Inappropriate $\mathrm{X}^{2} \mathrm{P}$ value

$\begin{array}{lll}96(82.05) & 21(17.95) & \\ 100(86.21) & 16(13.79) & < \\ & & 0.0001\end{array}$

$178(86.83) \quad 27(13.17)$

$16(55.17) \quad 13(44.83)$

$136(80.47) \quad 33(19.53)$

$254(85.23) \quad 44(14.77) \quad 0.183$

$333(84.52) \quad 61(15.48)$

$57(78.08) \quad 16(21.92) \quad 0.173$

$131(76.61) \quad 40(23.39)$

$152(88.89) \quad 19(11.11) \quad 0.007$

$107(85.60) \quad 18(14.40)$

$261(83.65) \quad 51(16.35) \quad 0.907$

$129(83.23) \quad 26(16.77)$

$107(83.59) \quad 21(16.41) \quad 0.977$

$283(83.48) \quad 56(16.52)$ 


\begin{tabular}{|llll|}
\hline No. of ANC attended & $30(61.22)$ & $19(38.78)$ & \\
\hline Once & $68(88.31)$ & $9(11.69)$ & \\
\hline Twice & $74(84.09)$ & $14(15.91)$ & 0.0001 \\
\hline Thrice & $69(84.15)$ & $13(15.85)$ & \\
\hline Four & $90(90.91)$ & $9(9.09)$ & \\
\hline Fifth-sixth & $59(81.94)$ & $13(18.06)$ & \\
\hline 7+ times & & & \\
\hline Gestational age & $64(67.37)$ & $31(32.63)$ & \\
\hline 16-20 weeks & $71(85.54)$ & $12(14.46)$ & \\
\hline $21-25$ weeks & $102(92.73)$ & $8(7.27)$ & \\
\hline 26-30 weeks & $87(88.78)$ & $11(11.22)$ & \\
\hline $31-35$ weeks & $66(81.48)$ & $15(18.52)$ & \\
\hline $36+$ weeks & & & \\
\hline
\end{tabular}

\section{Determinants of Appropriate Delivery of IPTp-SP}

Factors found to be significantly associated with appropriate delivery of IPTp-SP in the crude analysis were type of health facility, years of experience as an ANC staff, type of pregnant woman, number of ANC clinic sessions attended and gestational age of pregnant woman (Table 4). In the multivariable logistic regression analysis, type of facility, years of experience as an ANC staff and gestational age of pregnant woman were the predictors that remained significant with appropriate delivery of IPTp-SP. Regarding the type of facility, the faith-based hospital (HOSP 2) (aOR 0.68, 95\% Cl= 0.275-1.707), the health centres (aOR0.60, 95\% $\mathrm{Cl}=0.236-1.545)$ and CHPS compounds (aOR $0.12,95 \% \mathrm{Cl}=0.039-0.375$ ) had reduced odds of appropriate delivery compared to the district hospital (HOSP 1). Higher odds of appropriate delivery were found among ANC health workers with one to five years' experience (aOR 3.57, 95\% $\mathrm{Cl}=1.564-8.145)$ and more than five years' experience (aOR $3.08,95 \% \mathrm{Cl}=1.181$ 8.028). The odds of appropriate delivery of IPTp-SP increased with increasing gestational age, where pregnant women with gestational ages between 21 to 25 weeks had more than twice $(\mathrm{aOR}=2.33,95 \% \mathrm{Cl}=1.621-5.343)$ the odds of appropriate delivery, and those between 26-30 weeks had more than four times (aOR 4.64, 95\% Cl=1.80711.918) the odds of appropriate delivery compared to those between 16 to 20 weeks (Table 4).

\section{Table 4: Determinants of Appropriate Delivery}




\begin{tabular}{|c|c|c|c|c|c|c|}
\hline \multirow[t]{2}{*}{ Factors } & \multicolumn{3}{|c|}{ Unadjusted } & \multicolumn{3}{|c|}{ Adjusted } \\
\hline & OR & P-value & $95 \% \mathrm{Cl}$ & aOR & P-value & $95 \% \mathrm{Cl}$ \\
\hline \multicolumn{7}{|l|}{ Type of facility } \\
\hline District hospital & 1.00 & & & 1.00 & & \\
\hline Faith-based hospital & 1.37 & 0.387 & $0.6734-2.7756$ & 0.68 & 0.418 & $0.2758-1.7075$ \\
\hline Health Centres & 0.55 & 0.031 & 0.3175-0.9479 & 0.60 & 0.293 & $0.2362-1.5452$ \\
\hline CHPS Compounds & 0.27 & 0.003 & $0.1126-0.6432$ & 0.12 & 0.000 & $0.0397-0.3752$ \\
\hline \multicolumn{7}{|c|}{ ANC years of experience } \\
\hline$<$ one year & 1.00 & & & & & \\
\hline 1-5years & 2.44 & 0.003 & $1.3487-4.4242$ & 3.57 & 0.002 & $1.5647-8.1455$ \\
\hline$>5$ years & 1.8 & 0.056 & $0.9841-3.3476$ & 3.08 & 0.021 & $1.1818-8.0289$ \\
\hline \multicolumn{7}{|c|}{ Type of Pregnant woman } \\
\hline First time ANC & 1.00 & & & & & \\
\hline Continuing ANC & 3.93 & 0.000 & $2.0768-7.4407$ & - & - & - \\
\hline \multicolumn{7}{|l|}{ No. of ANC attended } \\
\hline Once & 1.00 & & & & & \\
\hline Twice & 4.78 & 0.001 & $1.9416-11.7928$ & - & - & - \\
\hline Thrice & 3.35 & 0.003 & $1.4888-7.5271$ & & & \\
\hline Four & 3.36 & 0.004 & $1.4724-7.6742$ & & & \\
\hline Fifth-sixth & 6.33 & 0.000 & $2.5897-15.4886$ & & & \\
\hline $7+$ times & 2.87 & 0.013 & $1.2519-6.5995$ & & & \\
\hline \multicolumn{7}{|l|}{ Gestational age } \\
\hline 16-20 weeks & 1.00 & & & 1.00 & & \\
\hline 21-25 weeks & 2.86 & 0.006 & $1.3576-6.0495$ & 2.33 & 0.044 & $1.0216-5.3434$ \\
\hline 26-30 weeks & 6.17 & 0.000 & $2.6722-14.2728$ & 4.64 & 0.001 & $1.8077-11.9186$ \\
\hline 31-35 weeks & 3.83 & 0.001 & $1.7919-8.1901$ & 2.73 & 0.046 & 1.0158-7.3888 \\
\hline $36+$ weeks & 2.13 & 0.036 & $1.0521-4.3170$ & 1.30 & 0.633 & $0.4404-3.8469$ \\
\hline
\end{tabular}

\section{Determining Eligibility of Pregnant Women for ITN}


Evidence of receipt of ITN by a pregnant woman during her current pregnancy at the ANC is indicated through its documentation in the MHRB of the woman. Pregnant women with no documentation of 'ITN given' in their MHRB was interpreted as ITN not yet received. Of the 657 ANC sessions observed, 127 of the pregnant women were first time ANC attendees with the remaining 530 being continuing clients. Seventy-nine percent (420) of the continuing clients had "ITN given" documented in their MHRB, with the remaining $21 \%$ (110), having no record of ITN given in their MHRB. Defining eligibility for ITN in this study as all pregnant women who have not yet received an ITN, the number of pregnant women eligible for the intervention was 237 as shown in figure 3.

\section{Assessment of ITN Delivery}

Using the study's defined delivery algorithm, ITN was appropriately delivered to $58.65 \%$ (139) of the 237 pregnant women who had not yet received an ITN prior to day of observation (Figure 4). Appropriate delivery was highest amongst first time ANC clients (114) than the continuing clients (25) (Table 4). While 21\% (21) of inappropriate delivery occurred during periods of stock out, the remaining $32 \%$ (77) of eligible women were not given ITN despite stock availability.

\section{Factors Associated with ITN Delivery}

Inappropriate delivery due to stock out was filtered out from the denominator of eligible pregnant women before computing factors influencing delivery of ITN. Pearson Chi-square analysis and Fishers Exact test were conducted to identify factors influencing delivery of ITN. The results showed that, the type of health worker $(p=0.018)$, type of pregnant woman $(p<0.0001)$, number of ANC clinic sessions attended $(p<0.0001)$, and gestational age of the pregnancy $(p<0.0001)$ were significantly associated with delivery of ITN $($ Table 5$)$. Appropriate delivery of ITN was highest among first time ANC clients (97.44\%), compared to continuing clients who have not yet received one (25.25\%). Similarly, delivery of ITN decreased with increasing number of ANC attendances (Table 5).

\section{Table 5: Factors Influencing ITN Delivery}




\begin{tabular}{|c|c|c|c|}
\hline \multirow[b]{2}{*}{ Factors } & \multicolumn{3}{|c|}{ ITN Delivery When in Stock ( $\mathrm{N}=216)$} \\
\hline & Appropriate & Inappropriate & $\mathrm{X}^{2}$ P-value \\
\hline \multicolumn{4}{|l|}{ Type of facility } \\
\hline District hospital & $49(71.01)$ & $20(28.99)$ & \\
\hline Faith-based hospital & $32(51.61)$ & $30(48.39)$ & 0.102 \\
\hline Health Centres & $50(67.57)$ & $24(32.43)$ & \\
\hline CHPS Compounds & $8(72.73)$ & $3(27.27)$ & \\
\hline \multicolumn{4}{|l|}{ Type of worker } \\
\hline Midwife & $106(60.57)$ & $69(39.43)$ & 0.018 \\
\hline Rotation nurse & $33(80.49)$ & $8(19.51)$ & \\
\hline \multicolumn{4}{|c|}{ ANC years of experience } \\
\hline$<$ one year & $61(64.21)$ & 34 (35.79) & \\
\hline 1-5years & $42(57.53)$ & $31(42.47)$ & 0.149 \\
\hline$>5$ years & $36(75.00)$ & $12(25.00)$ & \\
\hline \multicolumn{4}{|l|}{ MiP Training } \\
\hline Yes & $92(62.59)$ & $55(37.41)$ & 0.429 \\
\hline No & 47 (68.12) & $22(31.88)$ & \\
\hline \multicolumn{4}{|c|}{ Type of Pregnant woman } \\
\hline First time ANC & $114(97.44)$ & $3(2.56)$ & $<0.000$ \\
\hline Continuing ANC & $25(25.25)$ & $74(74.75)$ & \\
\hline \multicolumn{4}{|l|}{ No. of ANC attended } \\
\hline Once & $114(97.44)$ & $3(2.56)$ & \\
\hline Twice & $20(57.14)$ & $15(42.86)$ & \\
\hline Thrice & $2(9.09)$ & $20(90.91)$ & $<0.000$ \\
\hline Four & $1(7.69)$ & 12 (92.31) & \\
\hline Fifth-sixth & $1(9.09)$ & $10(90.91)$ & \\
\hline $7+$ times & $1(5.56)$ & $17(94.44)$ & \\
\hline \multicolumn{4}{|l|}{ Gestational age } \\
\hline$<15$ weeks & $64(95.52)$ & $3(4.48)$ & \\
\hline $16-20$ weeks & 35 (71.43) & $14(28.57)$ & \\
\hline 21-25 weeks & $14(58.33)$ & $10(41.67)$ & $<0.000$ \\
\hline
\end{tabular}




\begin{tabular}{|lll|}
\hline $26-30$ weeks & $17(54.84)$ & $14(45.16)$ \\
\hline $31-35$ weeks & $7(25.93)$ & $20(74.07)$ \\
\hline $36+$ weeks & $2(11.11)$ & $16(88.89)$ \\
\hline
\end{tabular}

\section{Discussion}

This study aimed at assessing the delivery of IPTp-SP and ITN to pregnant women attending ANC clinics in the Volta Region of Ghana, using the country's MiP policy directive as a guide. The use of structured observation checklists enabled the identification of the intricate delivery practices of the health workers. Furthermore, collection of data across three levels of health care delivery facilities enabled the identification of factors peculiar to each level of care and how it affects the delivery practices of the two preventive interventions. One factor was the observation of stock out of ITNs in the health centres. This level of facility had limited storage space, hence both pharmaceutical and non-pharmaceutical products were stored within the same space. The bulkiness of ITNs make it difficult to stock up for three months, the duration usually used in calculating quantities needed. Hence, the ITNs are stored in the district health directorates and supplied to the health centres intermittently. Delay in the district transporting requested quantities of ITNs leads to stock out, as most of these health centres do not have facility owned vehicles, therefore reliant on prompt supply by the district. Another health facility level factor that could affect the delivery of IPTp-SP is the unavailability of laboratory and ultrasound scan services in the health centres and CHPS compounds, although this was not statistically significant in this study. Starting IPTp-SP is dependent on knowing or ascertaining the gestational age of pregnant women, and in instances where the pregnant woman is uncertain about her gestational age due to poor recall of menstrual periods, the ultrasound scan machine can facilitate that (41). Facilities without laboratory capacity to perform G6PD tests to identify and exclude women who cannot take sulfa containing drugs like SP, could delay the administration of the first dose of IPTp-SP. Pregnant women may be referred to perform these tests elsewhere, and the cost associated with transport and payment of the services could be a barrier, hence delay the test and eventually starting the medication. A study conducted in a health centre in the Dangme West District of Ghana, revealed how pregnant women referred to other facilities for laboratory test discontinued ANC attendance altogether because they could not afford it (42).

Overall, inappropriate delivery of IPTp-SP was recorded in all the levels of health care delivery facilities, although it was highest in the health centres and the CHPS compounds. Intermittent stock out of SP, as observed during the data collection period in the health centres could account for the increased proportion of eligible pregnant women who did not receive the medication. That notwithstanding, in the absence of stock out in the hospitals, IPTp-SP was still not administered to some eligible pregnant women This highlights health worker factors influencing delivery of the intervention. This is not surprising as other studies have cited factors such as health worker forgetfulness, (43) poor health worker knowledge on delivery directives (44) as well as confusion among health workers over timing and dosing of SP (45) as reasons for missed opportunity to administer the medicine. One of the predictors of appropriate delivery of IPTp-SP in this study was years of experience of ANC staff. Workers with more than a years' experience were three times more likely to deliver IPTp-SP appropriately compared to those with less than a year's experience. A possible reason could be because ANC staff with less than a years' experience are newly graduated nurses who have not had the opportunity to attend any training to 
enlighten them on current approved delivery of IPTp-SP, hence less informed about delivery practices. This highlights the fact that the health training institutions may not have incorporated current policy directives on delivery of pertinent health services such as MiP in their training materials. Training of health workers especially the front line health workers makes them well informed and technically equipped to deliver MiP interventions effectively. However, these trainings are sometimes overlooked thereby affecting their perception about IPTp-SP and how to administer it to pregnant women (46) (47). A consequence of lack of training on IPTp-SP was reported by Rassi and colleagues (47) where health workers expressed doubts about the efficacy and safety of SP as an IPT because they do not understand why they were asked to discontinue SP for the treatment of clinical malaria, yet asked to retain it as an IPT. These workers indicated that they still use SP to treat symptomatic malaria because they feel it is more efficacious and better tolerated than other available antimalarial drugs (47). While a number of studies conducted in other African malaria endemic countries have reported the non-adherence to the DOT strategy of administering IPTp-SP with the most cited reason being unavailability of drinking water (44) (48), this study found the contrary. Almost all the pregnant women who were offered the medication by the ANC staff were given under DOT. Health workers in this context are adhering to the delivery strategy recommended. Pregnant women were seen to either bring their own water or buy a sachet of water to take the SP.

The study aimed at identifying gaps in the delivery of IPTp-SP and ITN that needs to be improved to realize optimal uptake of the interventions. Hence, prior to assessing delivery of the interventions, and classifying it as appropriate or inappropriate delivery, ineligible pregnant women as specified by the Ghana MiP policy directive were excluded to limit overestimation of inappropriate delivery as those women may not be given the IPTp-SP or ITN. Additionally, inappropriate delivery during periods of stock out were excluded from the sample before estimating predictors of correct delivery, in order not to confound the estimation of other predictor variables. These are strengths of the study, picked up from a recommendation by Webster and colleagues (45) who acknowledged it as a limitation in their study on assessing delivery effectiveness of IPTp-SP.

There are however a few limitations with this study design. One of such is the possibility of a Hawthorne's effect (49) on the ANC health staff. Acknowledging the fact that the observed staff may modify their behavior in response to their awareness of being observed, in this case gravitating towards 'appropriate behaviour', a considerable number of observations were conducted in the study facilities over a period of days and in some facility weeks. Therefore, these health workers are likely to revert back to their normal delivery practices, thereby enabling the study to assess the true delivery practices. It is important to note that, the Hawthorne's effect may be applicable in a context where the health workers are aware of the appropriate delivery practices but do not practice it, therefore, they would modify their behavior when they become conscious of observers. On the other hand, if the health workers are not aware of the appropriate delivery practices, being observed or not will not have any effect on their delivery practices. Another, limitation of this study could be the inability to capture all the delivery practices of the ANC staff by the observing research assistants. This is because the pace of delivery is not determined by the RAs, and this pace could be exceptionally swift on days where the ANC attendance is high. However, to curtail this limitation, community health nurses who are familiar with the health care delivery setup and more inclined to understand the clinical care and medical jargons were recruited as research assistants. Furthermore, rigorous training on data collection tools, coupled with extensive pre-test activities were carried out prior to the actual data collection to ensure that the RAs are able to collect the data accurately by looking and listening attentively to the delivery of care by ANC health staff. 


\section{Conclusions}

In conclusion, inappropriate delivery of IPTp-SP was observed across all three levels of the health care delivery facilities, and predictors of appropriate delivery were type of facility, years of experience of ANC staff and gestational age of pregnant woman. It should be noted that in the absence of stock as observed in mainly the hospitals, eligible pregnant women missed out on being given the medication. This highlights the facts that there could be other underlining factors which might be better elucidated through qualitative means of enquiry such as in-depth interview of health workers. Inappropriate delivery of ITN was also observed across all the health facilities included in this study.

\section{Abbreviations}

ANC Antenatal Care

CHPS Community-Based Health Planning Services

DHIMS District Health Management Information System

DOT Directly Observed Therapy

GDHS Ghana Demographic and Health Survey

GFR Genaral Fertility Rate

IPTp-SP Intermittent Preventive Treatment for Pregnant women using Sulfadoxine Pyrimethamine

ITNs Insecticide Treated Nets

MHRB Maternal Health Record Book

MiP Malaria in Pregnancy

P. Plasmodium

SP Sulfadoxine Pyrimethamine

SSA Sub-Saharan Africa

WHO World Health Organization

\section{Declarations}

\section{Ethical approval and consent to participate}

Ethical approval to conduct this study was obtained from the University of Health and Allied Sciences (UHAS) Ethics Board (UHAS-REC/A.I UI 17-18). Administrative letter of authorization to carry out the study in the Volta region and the study districts was obtained from the regional health directorate. All the respondents were provided with information about study and this entailed rationale for the study, the objectives, why they were 
selected and the approximate duration of the interviews or interaction. Written informed consent was obtained from all respondents who agreed and were willing to participate in the study. Informed consent was also obtained from parents or guardians of respondents who were less than 18 years old. All the data collection methods as well as analysis were carried out in accordance with the guidelines and regulations approved by the UHAS Ethics Board.

\section{Consent for Publication}

Not applicable

\section{Availability of data and materials}

The dataset analyzed for this study is available from the corresponding author on reasonable request.

\section{Competing interest}

The authors declare that they have no competing interest.

\section{Funding}

This work was supported through the MARCAD DELTAS Africa Initiative grant DEL-15-010. The DELTAS Africa Initiative is an independent funding scheme of the African Academy of Sciences (AAS)'s Alliance for Accelerating Excellence in Science in Africa (AESA) and supported by the New Partnership for Africa's Development Planning and Coordinating Agency (NEPAD Agency) with funding from the Wellcome Trust grant 107741/A/15/Z and the UK government. The views expressed in this publication are those of the author(s) and not necessarily those of AAS, NEPAD Agency, Wellcome Trust or the UK government.

\section{Authors contribution}

VFD designed and conceptualized the study with guidance from HT. VFD analyzed the data and wrote the paper. $\mathrm{HT}, \mathrm{PM}, \mathrm{JK}, \mathrm{WM}$, and ONV critically reviewed the manuscript for technical inputs. All the authors read and approved the final manuscript.

\section{Acknowledgement}

The authors wish to thank the pregnant women and the ANC health for their participation in the study. We would also like to thank the regional and district health directorates for their administrative support. Sincere gratitude also goes to the research assistants who diligently helped with data collection.

\section{References}

1. Naghavi M, Abajobir AA, Abbafati C, Abbas KM, Abd-Allah F, Abera SF, et al. Global, regional, and national age-sex specifc mortality for 264 causes of death, 1980-2016: A systematic analysis for the Global Burden of Disease Study 2016. Lancet. 2017;

2. WHO. World malaria report 2020- WHO [Internet]. 2020. Available from: https://www.who.int/publications/i/item/9789240015791 
3. WHO. World Malaria Report. https://www.who.int/malaria [Internet]. 2019. (accessed 15 Dec. 2019). Available from: https://www.who.int/publications-detail/world-malaria-report-2019

4. Takem E, D’Alessandro U. Malaria in pregnancy. Mediterr J Hematol Infect Dis. 2013;

5. Kourtis AP, Read JS, Jamieson DJ. Pregnancy and Infection. N Engl J Med [Internet]. 2014;370(23):2211-8. Available from: http://www.nejm.org/doi/10.1056/NEJMra1213566

6. Lindsay S, Ansell J, Selman C, Cox V, Hamilton K, Walraven G. Effect of pregnancy on exposure to malaria mosquitoes. Lancet. 2000;

7. Walker PGT, ter Kuile FO, Garske T, Menendez C, Ghani AC. Estimated risk of placental infection and low birthweight attributable to Plasmodium falciparum malaria in Africa in 2010: A modelling study. Lancet Glob Heal. 2014;

8. Falade $\mathrm{CO}$, Tongo OO, Ogunkunle OO, Orimadegun AE. Effects of malaria in pregnancy on newborn anthropometry. J Infect Dev Ctries. 2010;4(7):448-53.

9. Desai M, Dellicour S. Effects of malaria and its treatment in early pregnancy. Vol. 12, The Lancet Infectious Diseases. 2012. p. 359-60.

10. Adams Waldorf KM, McAdams RM. Influence of infection during pregnancy on fetal development. Reproduction [Internet]. 2013;146(5):R151-62. Available from:

http://www.ncbi.nlm.nih.gov/pubmed/23884862\%5Cnhttp://www.pubmedcentral.nih.gov/articlerender.fcgi? artid=PMC4060827

11. McLean ARD, Ataide R, Simpson JA, Beeson JG, Fowkes FJI. Malaria and immunity during pregnancy and postpartum: A tale of two species. Parasitology. 2015.

12. Balogun ST, Adeyanju OA, Adedeji AA, Fehintola FA. Predictors of asymptomatic malaria in pregnancy. Niger J Physiol Sci. 2011;26(2):179-83.

13. Ogbu GI, Aimakhu CO, Ajen Anzaku S, Ngwan S, Ogbu DA. Prevalence of malaria parasitaemia among asymptomatic women at booking visit in a tertiary hospital, Northcentral Nigeria. J Reprod Biol Heal. 2015;

14. Desai M, ter Kuile FO, Nosten F, McGready R, Asamoa K, Brabin B, et al. Epidemiology and burden of malaria in pregnancy. Vol. 7, Lancet Infectious Diseases. 2007. p. 93-104.

15. Idikwu, O.G., Anzaku, A.S., Adejoh OE. Burden of Anemia among Pregnant Women with Asymptomatic Malaria Parasitaemia at Booking Visit in Abuja, Nigeria [Internet]. Annals of International medical and Dental Research. 2016. p. 35-9. Available from:

http://aimdrjournal.com/pdf/Vol2Issue3_10_OA_Stephen_2_3_13.pdf

16. Steketee RW. Weighing in on malaria-attributable low birthweight in Africa. The Lancet Global Health. 2014.

17. WHO. World Malaria Report 2019. Geneva. World Malar Rep. 2019;

18. Hong R, Ruiz-Beltran M. Low birth weight as a risk factor for infant mortality in Egypt. East Mediterr Heal J. 2008;

19. World Health Organization. A strategic framework for malaria prevention and control during pregnancy in the African Region. Brazzav WHO Reg Off Africa [Internet]. 2004;1-39. Available from:

http://whqlibdoc.who.int/afro/2004/AFR_MAL_04.01.pdf

20. World Health Organization. Updated WHO Policy Recommendation ( October 2012) Intermittent Preventive Treatment of malaria in pregnancy using Sulfadoxine- Pyrimethamine ( PTp-SP). Glob Malar Program. 2012; (October):3-4. 
21. WHO. WHO Recommendation on Antenatal care for positive pregnancy experience. WHO Recomm Antenatal care Posit pregnancy Exp. 2016;

22. Feachem RG, Sabot OJ. An examination of the Global Fund at 5 years. Lancet. 2006.

23. Global Fund. The Global Fund [Internet]. 2020 [cited 2020 Nov 19]. Available from: https://www.theglobalfund.org/en/malaria/

24. Andrews KG, Lynch M, Eckert E, Gutman J. Missed opportunities to deliver intermittent preventive treatment for malaria to pregnant women 2003-2013: A systematic analysis of 58 household surveys in sub-Saharan Africa. Malar J. 2015;

25. WHO Global Malaria Programme. World Malaria Report 2016. World Health Organization. 2016.

26. Ghana Statistical Service. Ghana Demographic Health Survey. Ghana Stat Serv [Internet]. 2015;530. Available from: https://dhsprogram.com/pubs/pdf/FR307/FR307.pdf

27. Pell C, Straus L, Andrew EVW, Meñaca A, Pool R. Social and cultural factors affecting uptake of interventions for malaria in pregnancy in Africa: A systematic review of the qualitative research. PLoS One. 2011;6(7).

28. Hill J, Hoyt J, van Eijk AM, D’Mello-Guyett L, ter Kuile FO, Steketee R, et al. Factors Affecting the Delivery, Access, and Use of Interventions to Prevent Malaria in Pregnancy in Sub-Saharan Africa: A Systematic Review and Meta-Analysis. PLoS Med. 2013;

29. Ibrahim H, Maya ET, Issah K, Apanga PA, Bachan EG, Noora CL. Factors influencing uptake of intermittent preventive treatment of malaria in pregnancy using sulphadoxine pyrimethamine in sunyani municipality, Ghana. Pan Afr Med J. 2017;

30. Owusu-Boateng I, Anto F. Intermittent preventive treatment of malaria in pregnancy: a cross-sectional survey to assess uptake of the new sulfadoxine-pyrimethamine five dose policy in Ghana. Malar J. 2017;

31. Oppong FB, Gyaase S, Zandoh C, Nettey OEA, Amenga-Etego S, Anane EA, et al. Intermittent preventive treatment of pregnant women in Kintampo area of Ghana with sulphadoxine-pyrimethamine (SP): Trends spanning 2011 and 2015. BMJ Open. 2019.

32. Ghana Statistical Service. Agotime ziope district [Internet]. 2014. Available from: http://www2.statsghana.gov.gh/docfiles/2010_District_Report/VOLTA/AGORTIME\%25ZIOPE.pdf

33. Ghana Statistical Service (GSS). South tongu district. 2014; Available from: www.statsghana.gov.gh.

34. Glenn D Isreal. Determining Sample Size. 2003; Available from: https://www.tarleton.edu/academicassessment/documents/Samplesize.pdf

35. National Malaria Control Programme. National Malaria Control Programme (Ghana). 2016.

36. Ghana Statistical Service. Ghana demographic and health survey 2014. Ghana demographic and health survey 2014. 2014. 1-506 p.

37. Azizi SC, Chongwe G, Chipukuma H, Jacobs C, Zgambo J, Michelo C. Uptake of intermittent preventive treatment for malaria during pregnancy with Sulphadoxine-Pyrimethamine (IPTp-SP) among postpartum women in Zomba District, Malawi: A cross-sectional study. BMC Pregnancy Childbirth. 2018;

38. Daniel P. Schuster WJP. Translational and Experimental Clinical Research [Internet]. 2005. p. 490. Available from: https://books.google.com/books?id=C7pZftbl0ZMC\&pgis=1

39. NMCP. Guidelines on malaria in Pregnancy. Accra-Ghana; 2014.

40. Kearns Annie, Hurst Taylor, Caglia Jacquelyn LA. Focused antenatal care in Tanzania. women Heal Initiat. 2014; 
41. Brentlinger PE, Dgedge M, Correia MAC, Rojas AJB, Saúte F, Gimbel-Sherr KH, et al. Intermittent preventive treatment of malaria during pregnancy in central Mozambique. Bulletin of the World Health Organization. 2007.

42. Ansah-Ofei A, Mwini-Nyaledzigbor PP, Affram CK. Improving the quality of life of pregnant women using the intermittent preventive treatment with sulphadoxine pyrimethamine (IPT-SP): experiences from Dangme West District, Ghana. J Ghana Sci Assoc. 2011;

43. Ashwood-Smith H, Coombes Y, Kaimila N, Bokosi M, Lungu K. Availability and use of sulphadoxinepyrimethamine (SP) in pregnancy in Blantyre District: A Safe Motherhood and BIMI Joint Survey. Malawi Med J - J Med Assoc Malawi. 2002;

44. Onoka CA, Hanson K, Onwujekwe OE. Low coverage of intermittent preventive treatment for malaria in pregnancy in Nigeria: Demand-side influences. Malar J. 2012;

45. Webster J, Kayentao K, Bruce J, Diawara SI, Abathina A, Haiballa A, et al. Prevention of Malaria in Pregnancy with Intermittent Preventive Treatment and Insecticide Treated Nets in Mali: A Quantitative Health Systems Effectiveness Analysis. 2013;8(6).

46. Onwujekwe OC, Soremekun RO, Uzochukwu B, Shu E, Onwujekwe O. Patterns of case management and chemoprevention for malaria-in-pregnancy by public and private sector health providers in Enugu state, Nigeria. BMC Res Notes. 2012;

47. Rassi C, Graham K, Mufubenga P, King R, Meier J, Gudoi SS. Assessing supply-side barriers to uptake of intermittent preventive treatment for malaria in pregnancy: A qualitative study and document and record review in two regions of Uganda. Malaria Journal. 2016.

48. Mubyazi GM, Bloch P, Byskov J, Magnussen P, Bygbjerg IC, Hansen KS. Supply-related drivers of staff motivation for providing intermittent preventive treatment of malaria during pregnancy in Tanzania: Evidence from two rural districts. Malar J. 2012;

49. Merrett F. Reflections on the Hawthorne effect. Educational Psychology. 2006.

\section{Figures}




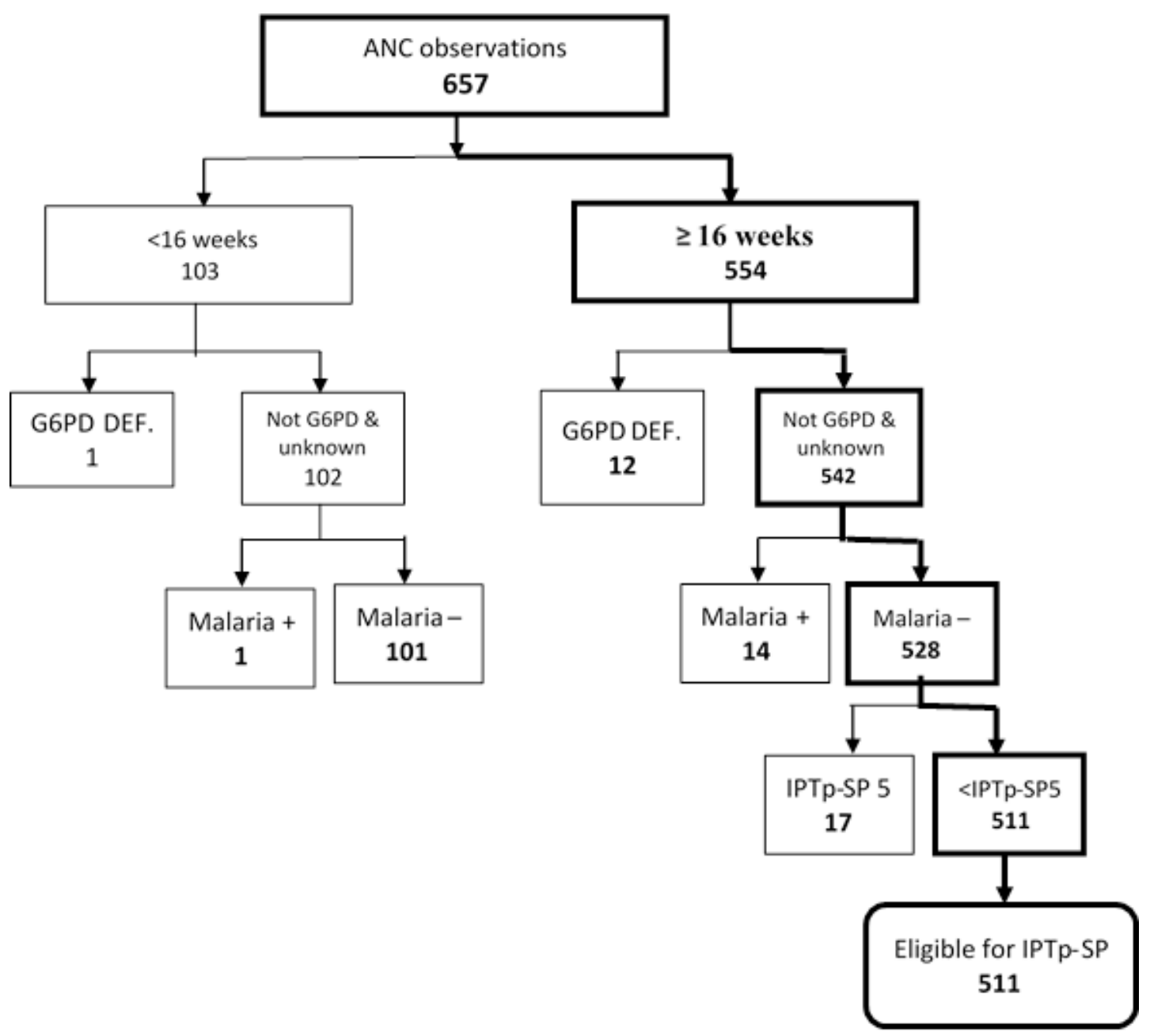

Figure 1

Determining Eligibility of Pregnant Women for IPTp-SP 


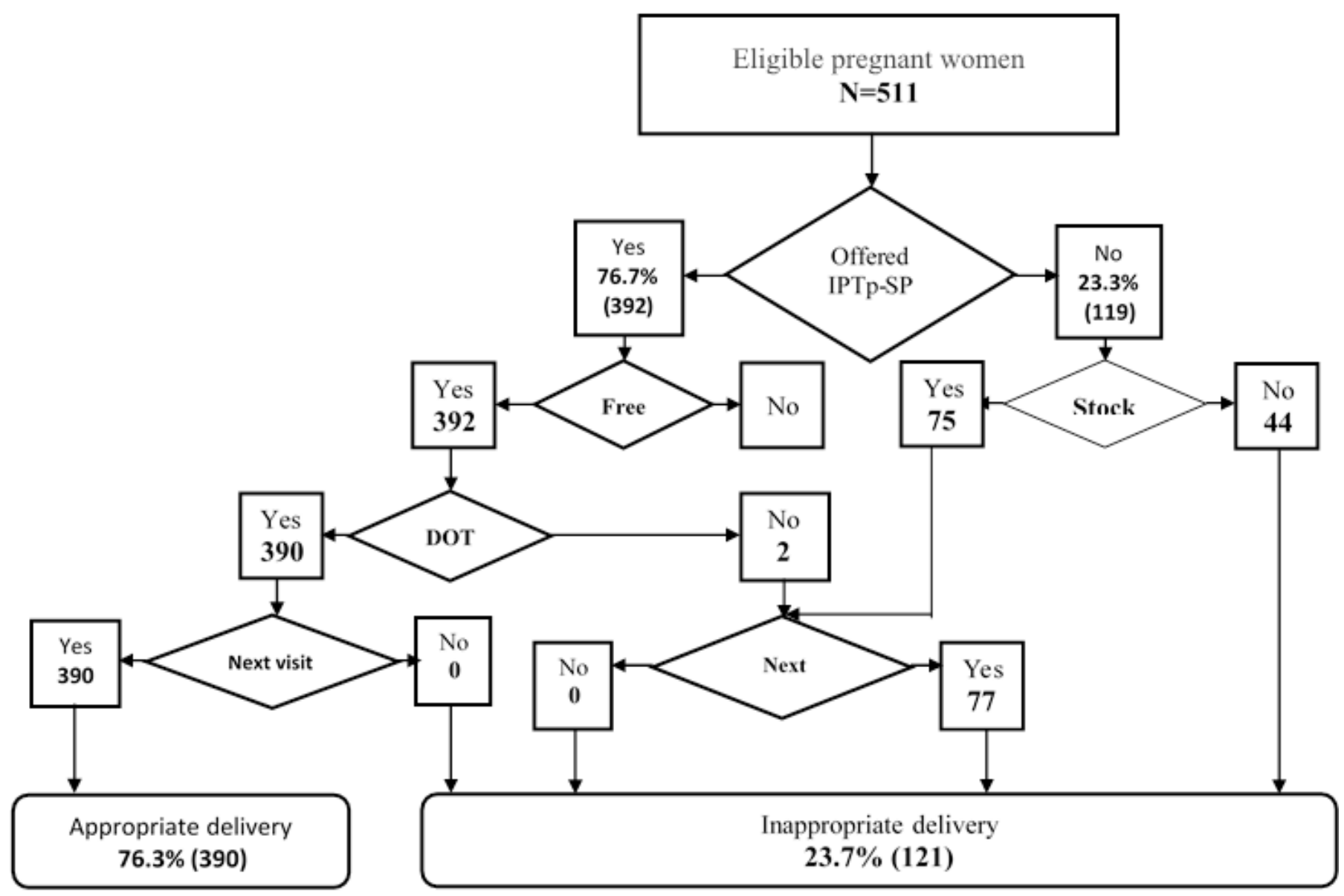

Figure 2

IPTp-SP Delivery Algorithm

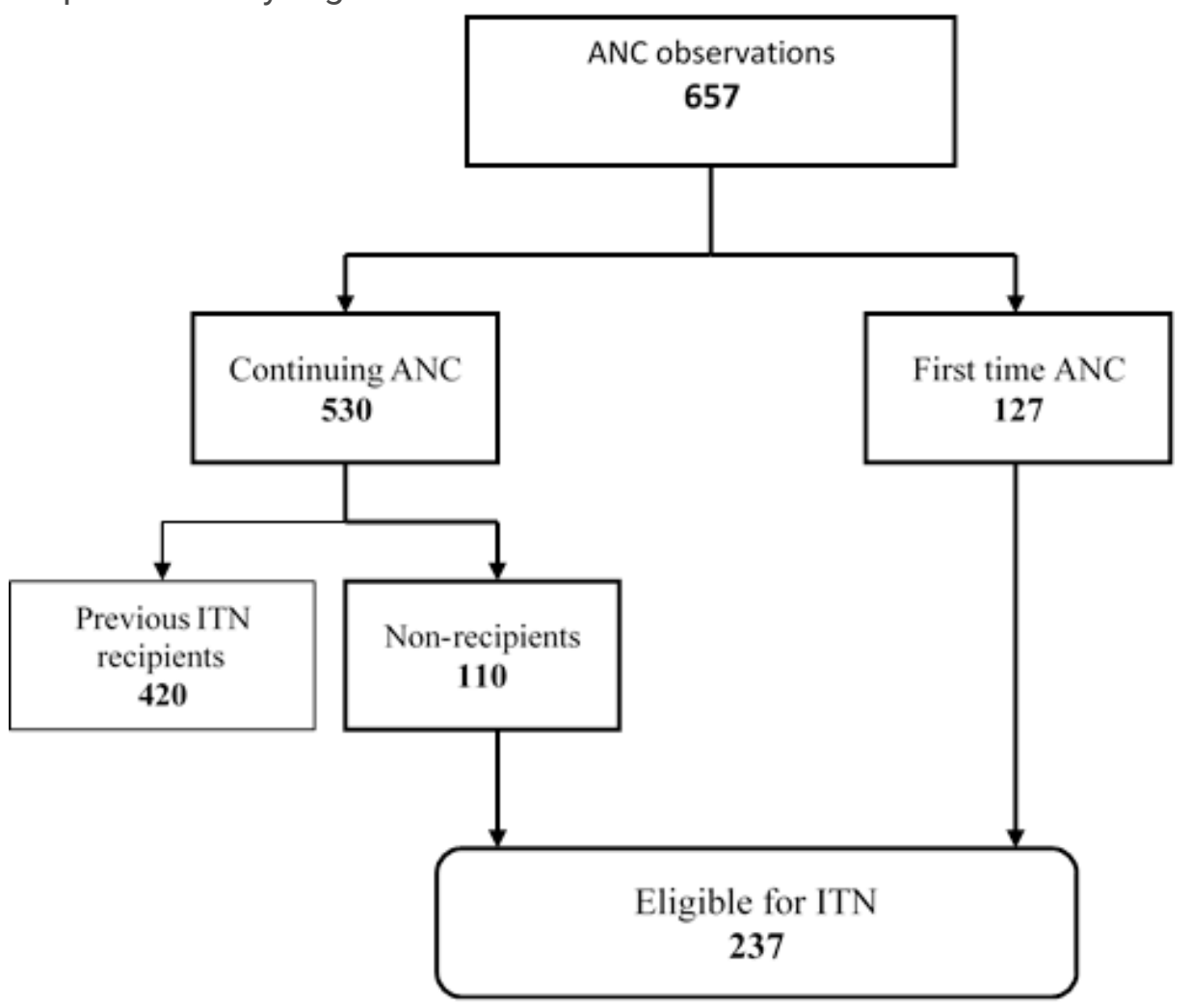


Figure 3

Determining eligibility of pregnant women for ITN

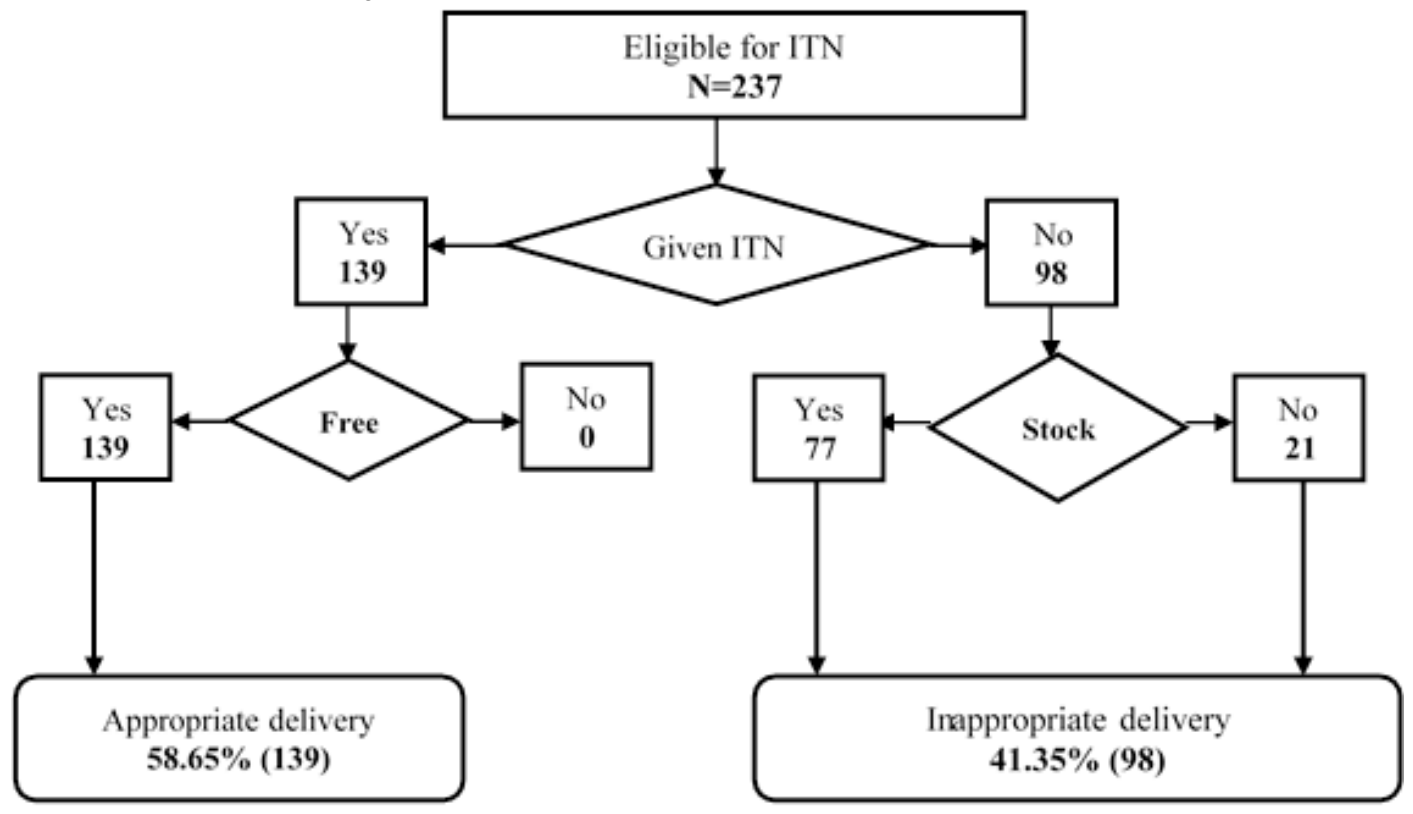

Figure 4

ITN Delivery 